\title{
Local Minimum Characterization of Interactingtime Series Data: Application in Stability Andstabilization
}

\author{
E.N. Ekaka-A,C.C. Wokocha,N.M. Nafo,E.H. Amadi \\ Department Of Mathematics And Computer Science, Rivers State University Of Science And Technology, Port \\ Harcourt, Nigeria, \\ Department Of Crop And Soil Science, Faculty Of Agriculture, University Of Port Harcourt, Nigeria
}

\begin{abstract}
The dynamics of the best-fit mathematical model which can beused to describe the interaction between cowpea and groundnut is an importantscientific problem. In this study, we propose to use the popular Euclideannorm or 2-norm cost function method to select the best-fit model parameters. Having selected the bestfit mathematical model, we intend to determine theimportant model parameters of this interacting system. The application ofstability and stabilization of this proposed mathematical model will studiedand presented. Our novel results which we have not seen elsewhere will bepresented and discussed with the expectation of providing further insightsabout these crop growth time series data.
\end{abstract}

Key words and phrases: Best-fit parameters, agricultural data, 2-norm, stability, stabilization.

\section{Introduction}

This present study is based on the current data which were collected by twoexperts in microbiology who are working in the Niger Delta Region of Nigeria ([1]).Extensive literatures which relate to plant-plant interactions, modellingbiolog-ical interacting populations, equations of determinate growth and plant growthanalysis are ([3]; [4]; [5]; [6]; [7]; [8]; [9]; [10]; [11]; [12]). Given these data on the growth of legumes ([1]), it is a challenging scientific problem to construct a mathematical model which describes the dynamics of any twointeracting legumes within an uncontaminated environmental setting. Attemptingto develop these distinct nonlinear model equations will provide crop scientists withuseful information such as the growth rates for two types of legumes, the intraspecific coefficients of legumes and their mass law action or interspecific coefficients asis popularly known in mathematical biology and molecular physics. The information about the doubling times of the two interacting legumes is an important insightwhich will assist further research in the stabilization of the mathematical model ofinteracting legumes and other land equivalent ratio studies in livelihood analysisand possibly about the numerical simulation of mutualism due to increasing sealevel rise from a competition interaction model between two legumes in severelyaffected communities in the Niger Delta Region of Nigeria.For the purpose of our present study, we propose to utilize the penalty-functionselection methods to select the best-fit model parameters from these uncontaminated agricultural data. As far as we know, this novel innovative numerical simulation technique has not been previously implemented computationally to tacklethis interesting proposed scientific problem in the biological sciences.

\section{Methodology}

In this study, we will focus on the application of the three popular mathematicalnorms ([2]) to select the best-fit parameters which are expected to characterize thedynamics of the interaction between two legumes such as the cowpea and groundnutover the growth season in weeks. The main thrust of this method concerns howwell to select the parameters which will provide the best-fit between the provideddata and our simulated data. We will deduce this characteristic of these dataif we can successfully find the local minimum from a sequence of the 1-norm, 2-norm and infinity-norm monotonic values. Without a detailed familiar simplicationabout the notions of mathematical norms, we will simply present our calculationssubsequently which we have obtained in the context of selecting the best-fit modelparameters from some uncontaminated data of cowpea and groundnut.The core part of our method depends on an appropriate set up of a single initialvalue logistic model with only two parameters namely the intrinsic growth rate forthe growing cowpea as well as the two similar parameters for the growing groundnutover a growing period in weeks. The data of [1] were collected every two weeks. Ourproposed simulation technique is constructed using the logic that if the beginningof the first week starts on the first day, then the seond week will start on day 8 . Inour context of the length of the growing period, subsequently after every two weeksthe growth data will be obtained by simulation on day 22 , followed by day 36 , thenon day 50 , on day 64 and on day 78 . By this proven procedure, our simulateddata and the provided data will have six data points. Therefore, we can nowmeasure the error between the provided data and our simulated data provided thatwe can successfully find the local minimum from the penalty-functions sequence ofmonotonic precise values. 
In the following sequence of results, our aim is to find the best-fit model param-eters using the three popular mathematical norms. The estimated daily growthrate for the cowpea data is 0.0225 . This value is calculated by dividing the seconddata value of 1.81 by the first data value of 1.32 and taking the logarithm to basee. This estimated growth rate of 0.315695108679455 is called the weekly growthrate. Our expected daily growth rate of 0.022549650619961 can now be obtained bydividing this weekly growth rate by 14 since the data provided were collected everytwo weeks. For the purpose of this study, we prefer to consider only the estimateddaily growth rate which is approximated to only 4 decimal places.

From Table 1, we observe that the local minimum for the 2-norm is 0.4691 . Asimilar analysis was conducted for the groundnut data to find the local minimumand hence deduce its corresponding logistic model. We have found a smaller local minimum value of 0.4673 using the 2-norm selection of best-fit parameters which correspond to the two values of the intraspecificcoefficients 0.007500 and 0.007475 when the steadystate values are 3.00 and 3.01 .

Therefore, the 2-norm penalty function selected interspecific interaction model between cowpea and groundnut under a realistic assumed value of the interspecificcoefficient is

Examples Calculation of the local minimum

no a b ss 2 - norm

10.02250 .01321 .711 .4881

20.02250 .01151 .961 .1881

30.02250 .01022 .210 .9271

40.02250 .00912 .460 .6940

50.02250 .00832 .710 .5419

60.02250 .00762 .960 .4691

70.02250 .00703 .210 .5010

80.02250 .00653 .460 .6046

90.02250 .00613 .710 .7288

100.02250 .00573 .960 .8824

Table 1. Calculation of the local minimum for the Cowpea data:

the notation ss stands for the steady state

$\mathrm{dG}(\mathrm{t}) / \mathrm{dt}=\mathrm{G}(\mathrm{t})(0.0446-0.0121 \mathrm{G}(\mathrm{t})-0.002 \mathrm{C}(\mathrm{t}))$.

where the initial biomasses are chosen appropriately.

Application in Stability

In this section, we are interested to study the stability behaviour of the steady-state solutions which define the dynamics of the above derived Lotka-Volterra sys-tem of continuous nonlinear first order ordinary differential equations.

Without loss of generality, following the mathematical theories of steady-state

solutions and stability, our numerical mathematical analysis of the above Lotka-Volterrra system is reported as follows:

Examples Calculation of the stability behaviour

no steady-state solutions $\lambda$

1

2 Each Type of Stability

$1(0,0) 0.02250 .0446$ Unstable

$2(0,3.686)-0.04460 .0078$ Unstable

$3(3,0)-0.02250 .0386$ Unstable

$4(1.134,3.50)-0.0076-0.043$ Stable

Table 2. Numerical calculations of the steady-state solutions and

their stability

It is very clear from this study that our best-fit mathematical model has only

four steady-state solutions out of which three are unstable ( and will require further

stabilizations) while the only unique positive co-existence steady-state solution is

said to be stable. The trivial steady-state solution implies that the two interacting

populations of cowpea and groundnut will go into the ecological risk of extinction

while the semi-trivial steady-state solutions will imply the theory of competitive

exclusion, that is, one population will survive at its carrying capacity while the

second population will be driven into extinction.4. Application in Stabilization

In this section, we are interested in studying the extent of stabilizing the unstable 
steady-state solutions which we have determined in the last section. Our results will be presented for the unstable trivial steady-state solution and for the unstable semi-trivial steady-state solutions. These results clearly compliment the theory of stabilizing a mathematical model of population system ([13]) but differ in the sense that the stabilization of these unstable steady-state solutions is systematically conducted for a varying final time. The number of loops, that is, the number of repeated simulations which guarantees the stabilization for a given Tfinal and the step length $\mathrm{k}$ can be calculated using the simulation formula in which the specified value of the Tfinal is divided by the step length value of 0.01 . As such for a given simulation run, the number of loops can be calculated. It is worth mentioning that there is a perfect correlation between the choosen values of the Tfinal and the number of loops for a fixed step length $\mathrm{k}$ of 0.01 . We also observed that for the range of Tfinal values between 5 days and 100 days, stabilization of the trivial unstable steady-state solution is delayed while near stabilization will occur when the Tfinal takes values between 200 days and 300 days. Full stabilization will occur when Tfinal takes values between 400 days and 1000 days.

4.1. Stabilization of the unstable trivial steady-state solution. For a fixed step length value of 0.01 and varying values of the Tfinal in the unit of days, we are interested to find out if the unstable trivial steady-state solution can exhibit qualitative characteristics of delayed stabilization, near stabilization and full stabi-lization. Our novel contributions in this scenario are displayed next for the starting biomasses of cowpea and groundnut having the values of 1.5 grams and 1.4 grams. Examples Calculation of the convergence point no Tfinal N1e N2e 150.248770707522020 .180209205013358

2100.2055251240419500 .148683265740912

3150.1707403047318880 .123519836168339

4200.1420537858676210 .102767738484468

5250.1183318003158510 .085606790411431

6300.0986712142868340 .071383773075409

7350.0823464280100540 .059573852811441

8400.0687705670263680 .049752522513139

9450.0574662742047690 .041574482640261

10500.0480434204482140 .034757523617704

11600.0336180981335400 .024321464556882

12700.0235508031355660 .017038190666189

13800.0165113638708080 .011945422572436

14900.0115824737923700 .008379545586418

151000.0081280924401190 .005880417712843

162000.0002370965549520 .000171532217878

173000.0000069301732960 .000005013772002

184000.0000002025763330 .000000146557886

195000.0000000059215320 .000000004284050

2010000.0000000000000000 .000000000000000

Table 3. Calculation of the stabilization values for the unstable trivial steady-state solution

The main result which we have obtained from this table is the fact that the unstable trivial steady-state solution can be fully stabilized when the Tfinal is 1000 days or approximately two and half years. Although this result is mathematically tractable because as the time variable approaches infinity ([13]), N1e

converges to

zero and $\mathrm{N} 2 \mathrm{e}$

converges to zero, however this result does not provide meaningful sci-entific insight. On the other hand, the delayed and near stabilization scenarios can

provide useful information for growing two legumes such as cowpea and groundnut and hence enhance their productions. These insights are capable of guiding the legume farmers in terms of the timing of planting and the timing of harvesting. 
4.2. Stabilization of the unstable semi trivial steady-state solution $(0,3.686)$.

For a fixed step length value of 0.01 and varying values of the Tfinal in the unit of days, we are interested to find out if the unstable semi-trivial steady-state solution $(0,3.686)$ can similarly exhibit qualitative characteristics of delayed stabilization, near stabilization and full stabilization. Our novel contributions in this scenario are displayed next for the starting biomasses of cowpea and groundnut having the values of 1.5 grams and 1.4 grams.

Examples Calculation of the convergence point no Tfinal N1e N2e

150.6920018877533130 .664624424891831

2100.6783792560285120 .721628597491191

3150.6636907154348230 .787055725688576

4200.6471234544115450 .860788751410527

5250.6285714492144570 .943271757752760

6300.6079647707491191 .034786913533901

7350.5852809449697971 .135401407696008

8400.5605564803879131 .244917553499621

9450.5338965503326771 .362831894897302

10500.5054812065149831 .488310482449745

11600.4444797209003821 .75698906488650

12700.3803767613165552 .038304210991296

13800.3166254553895492 .317035597442157

14900.2565978352827022 .578537850872500

151000.2029117539099922 .811634643751604

162000.0097155407112913 .644373053901081

173000.0003636936202343 .684440657470661

184000.0000247629741613 .685892372365511

195000.0000126268471383 .685944353536691

2010000.0000121763529343 .685946283082031

Table 4. Calculation of the stabilization values for the unstable semi-trivial steady-state solution $(0,3.686)$

In this scenario, we can consider the unstable semi-trivial steady-state solution $(0,3.686)$ to be relatively fully stabilized when the final time is 1000 days. The same semi trivial steady-state solution has shown qualitative characteristics of delayed stabilization and near stabilization.

4.3. Stabilization of the unstable semi trivial steady-state solution $(3,0)$

For a fixed step length value of 0.01 and varying values of the Tfinal in the unitof days, we are interested to find out if the unstable semi-trivial steady-state so-lution $(3,0)$ can similarly exhibit qualitative characteristics of delayed stabiliza-tion, near stabilization and full stabilization. Our novel contributions in this scenario are displayed next for the starting biomasses of cowpea and groundnut having the values of 4.8 grams and 1.5 grams. In this scenario, when the fi-nal time is 5 days, the steady-state solution $(\mathrm{N} 1 \mathrm{e}, \mathrm{N}$

2e) converges to the point

(3.222786822990921, 0.042295457256194) while whenthe final time is 10 days, the steady-state solution (N1e,N

$2 \mathrm{e}$

) converges to the point (3.180492351348109, 0.033515607993592).

Both delayed and near stabilizations continue to exist until this unstable semi-trivial steady-state solution becomes fully stabilized when the final time is 1000 days as the steady-state solution (N1e,N

$2 \mathrm{e}$

) converges to the point (3.000000000000893, 0.000000000000162).

5. Concluding Remarks and Further Research

In this study, we have used the technique of local minimum for 2-norm penalty function to select the best-fit model parameters which form the best-fit candidate model. The stability of this model was studied. The qualitative behaviour of the steady-state solutions consistently display the ecological theory of extinction, 
competitive exclusion and co-existence. For the unstable steady-state solutions, we have developed a controller using the Riccatti formulation to stabilize them over a variation of the final time in the unit of days. We will expect these novel contributions to provide insights for the growth and production of legumes which will ultimately enhance food production security in Nigeria.

\section{References}

[1] M.A. Ekpo and A.J. Nkanang, Nitrogen fixing capacity of legumes and their Rhizosphereal microflora in diesel oil polluted soil in the tropics, Journal of Petroleum and Gas Engineering 1(4), (2010), pp. 76-83.

[2] E.N. Ekaka-a, Computational and Mathematical Modelling of Plant Species Interactions in a Harsh Climate, PhD Thesis, Department of Mathematics, The University of Liverpool and The University of Chester, United Kingdom, 2009.

[3] C. Damgaard, Evolutionary Ecology of Plant-Plant Interactions-An Empirical ModellingAp-proach, Aarthus University Press, 2004.

[4] E. Renshaw, Modelling Biological Populations in Space and Time, Cambridge University Press, 1991

[5] M. Kot, Elements of Mathematical Ecology, Cambridge University Press, 2001

[6] J.D. Murray, Mathematical Biology, 2nd Edition Springer Berlin, 1993.

[7] D. Tilman, Dynamics and Structure of Plant Communities, Princeton University Press, 1988

[8] T.O. Ibia, M.A. Ekpoand L.D. Inyang, Soil Characterisation, Plant Diseases and Microbial Survey in Gas Flaring Community in Nigeria, World J. Biotechnol. 3, (2002), pp. 443-453

[9] R. Hunt, Studies in Biology, no. 96: Plant Growth Analysis, London: Edward Arnold (Pub-lishers) Limited, 1981

[10] J. Goudriaan, J.L Monteith, A mathematical function for crop growth based on light inter-ception and leaf area expansion, Annals of Botany 66, (1990), pp. 695-701.

[11] J. Goudriaan, H.H. van Laar, Modelling potential crop growth processes, Dordrecht: Kluwer Academic Publishers, 1994

[12] X. Yin, J. Goudriaan, E.A. Lantinga, J. Vos and H.J. Spiertz, A Flexible Sigmoid Function of Determinate Growth, Annals of Botany 91, (2003), pp. 361-371.

[13] Yubin Yan, Enu-Obari N. Ekaka-a, Stabilizing a mathematical model of population system, Journal of the Franklin Institute 348, (2011), pp. 2744-2758 